\title{
Impact of drug and equipment preparation on pre-hospital emergency Anaesthesia (PHEA) procedural time, error rate and cognitive load
}

\author{
Paul Swinton ${ }^{1,2^{*}}$ D, Alasdair R. Corfield ${ }^{3}$, Chris Moultrie ${ }^{3,4}$, David Percival ${ }^{1}$, Jeffrey Proctor ${ }^{1}$, Neil Sinclair ${ }^{5}$
} and Zane B. Perkins ${ }^{6}$

\begin{abstract}
Background: We examined the effect of advanced preparation and organisation of equipment and drugs for Pre-hospital Emergency Anaesthesia (PHEA) and tracheal intubation on procedural time, error rates, and cognitive load.

Methods: This study was a randomised, controlled experiment with a crossover design. Clinical teams (physician and paramedic) from the Emergency Medical Retrieval Service and the Scottish Air Ambulance Division were randomised to perform a standardised pre-hospital clinical simulation using either unprepared (standard practice) or pre-prepared (experimental method) PHEA equipment and drugs. Following a two-week washout period, each team performed the corresponding simulation. The primary outcome was intervention time. Secondary outcomes were safety-related incidents and errors, and degree of cognitive load.

Results: In total 23 experiments were completed, 12 using experimental method and 11 using standard practice. Time required to perform PHEA using the experimental method was significantly shorter than with standard practice $(11,45$ versus 20:59) minutes: seconds; $p=<0.001)$. The experimental method also significantly reduced procedural errors ( 0 versus $9, p=0.007$ ) and the cognitive load experienced by the intubator assistant ( 41.9 versus $68.7 \mathrm{~mm}, p=0.006$ ).
\end{abstract}

Conclusions: Pre-preparation of PHEA equipment and drugs resulted in safer performance of PHEA and has the potential to reduce on-scene time by up to a third.

Keywords: Airway, Intubation, Emergency, Patient safety / safety, Human error, Drug preparation, Risk management, Human factors

\section{Background}

The primary purpose of an ambulance service is to provide rapid access to emergency care. This involves prompt, effective pre-hospital care and rapid transport to hospital.

Pre-hospital interventions that delay transport to hospital may worsen outcome [1]. For effective pre-hospital care, it is therefore important that the likely benefit of any

\footnotetext{
* Correspondence: pswinton@nhs.net

1Emergency Medical Retrieval Service, ScotSTAR, Scottish Ambulance Service, Glasgow, UK

${ }^{2}$ Scottish Air Ambulance Division, Scottish Ambulance Service, Glasgow, UK Full list of author information is available at the end of the article
}

intervention is weighed up against potential risks, including delayed transport to hospital.

Pre-hospital Emergency Anaesthesia (PHEA) with oral tracheal intubation is the technique of choice to manage critically ill or injured patients who cannot maintain their airway or achieve adequate ventilation [2]. While a potentially life-saving intervention in this group of patients, PHEA is associated with significant risks and is a recognised cause of prolonged on-scene times [1-5]. Most PHEA complications are predictable, and risk can be significantly reduced with appropriate preparation [2]. However, it is this preparation step that accounts for the majority of procedural time. A particular challenge

(c) The Author(s). 2018 Open Access This article is distributed under the terms of the Creative Commons Attribution 4.0 International License (http://creativecommons.org/licenses/by/4.0/), which permits unrestricted use, distribution, and reproduction in any medium, provided you give appropriate credit to the original author(s) and the source, provide a link to the Creative Commons license, and indicate if changes were made. The Creative Commons Public Domain Dedication waiver (http://creativecommons.org/publicdomain/zero/1.0/) applies to the data made available in this article, unless otherwise stated. 
in improving the overall benefit of PHEA is to reduce the time penalty of the procedure while ensuring that the highest safety standards are achieved [2].

In the UK, thirty pre-hospital services provide PHEA, and perform approximately 1600 PHEA procedures a year [6]. There is some variability between services with regard to the amount of PHEA preparation that is done prior to tasking, and the amount done on-scene. The busiest services pre-prepare equipment and drugs prior to tasking, while the majority of services perform this step on-scene.

The aim of this study was to evaluate the effect of pre-prepared equipment and drugs, on PHEA procedure time and safety. In addition, we assessed the effect that pre-preparation had on the cognitive load of clinicians. We hypothesised that the use of pre-prepared PHEA equipment and drugs could reduce procedural time and risk, thus improving the overall benefit of the intervention.

\section{Methods}

\section{Study design}

This was a randomised, controlled simulation experiment with a crossover design. The study design is presented in Fig. 1 with the full study protocol showing more detail [see Additional file 1].

Experiments were conducted between 04 January and 01 March 2017. The study was reviewed and approved by Queen Mary, University of London Research Ethics Committee (QMREC1839a), Greater Glasgow and Clyde Clinical Research \& Development committee (GN16AE762), and the Scottish Ambulance Service. Written informed consent was obtained from participants.

\section{Participants and setting}

The study was undertaken at Scotland's national Specialist Transport and Retrieval service (ScotSTAR). The service exists to provide a safe and dedicated transport and retrieval service to the 5.5 million population of Scotland $[7,8]$.

The Emergency Medical Retrieval Service (EMRS), ScotSTAR's adult retrieval service is a physician-led team delivering a primary pre-hospital response, working with the ambulance service to provide pre-hospital critical care at the scene of incidents. The EMRS team comprises a consultant physician, and a retrieval practitioner.

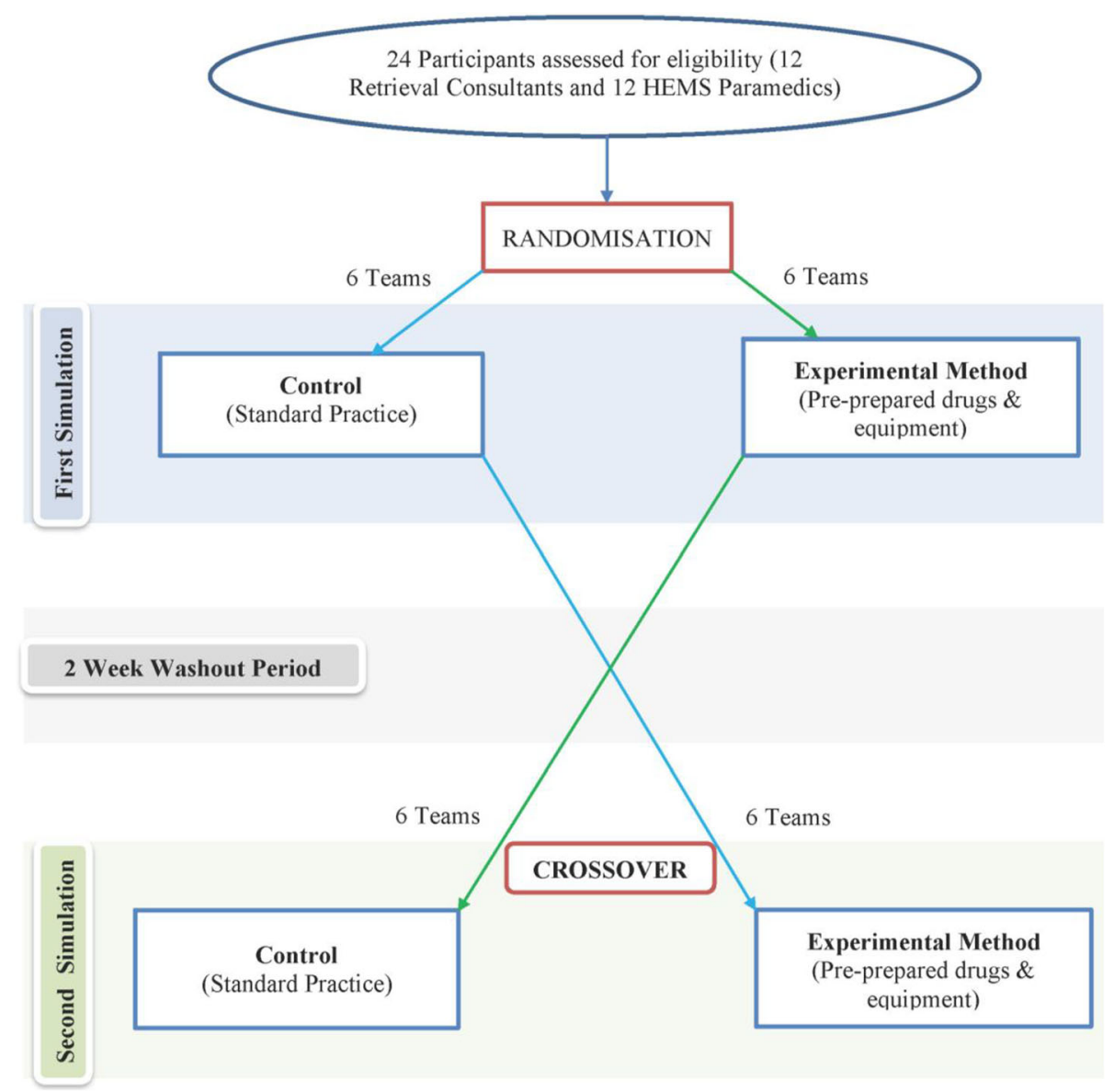

Fig. 1 Study Scheme Diagram 
When deployed by air, the EMRS team delivers PHEA with a Helicopter Emergency Services (HEMS) Paramedic in the role of intubator assistant.

The service has strict clinical governance procedures, which includes an intense training program prior to independent pre-hospital practice; adherence to SOPs that govern all aspects of pre-hospital practice, including the delivery of PHEA; and regular simulation training in the application of these procedures.

Eligible participants are experts in pre-hospital care, and perform PHEA as part of their normal working practice. Consultant retrieval physicians were recruited from the Emergency Medical Retrieval Service (EMRS), ScotSTAR's adult retrieval service. As this study was conducted within the Greater Glasgow and Clyde NHS board (NHS GG\&C) only consultant physicians currently working for the Emergency Medical Retrieval Service and employed by NHS GG\&C were permitted to participate.

Paramedics were recruited from the Scottish Ambulance Service pool of HEMS Paramedics.

Consultant retrieval physicians were eligible for enrolment if they were: 1) currently working with EMRS and employed by NHS GG\&C, 2) had > 6 months' experience as a retrieval physician, and 3) had been assessed by EMRS to be competent and current at PHEA.

HEMS paramedics were eligible for enrolment if they 1) were currently working for the SAS air ambulance division alongside EMRS, 2) had $>6$ months' experience as a retrieval paramedic, 3 ) had $>8$ years' experience as a paramedic, 4) had completed a recognised PHEA course [9], and 5) were assessed by EMRS to be competent and current at assisting PHEA.

All eligible participants were emailed an invitation to participate in the study. Willing participants were randomized using a computerised random number generator to identify which eligible participants would be enrolled into the study.

\section{Randomisation and allocation concealment}

A third party, not involved with enrolment and unaware of the study outcomes, used a computerised random number generator to block randomised participants into 12 two-person (physician /paramedic) teams. In addition, computer batch randomisation was used to establish the first simulation that would be undertaken by the team (standard practice or experimental method). Clinical teams were randomised to either the standard practice or experimental arm. Following a two-week wash-out period, the same clinical teams performed the corresponding simulation. Before each simulation, teams received a standardised briefing, including review of the services standard operating procedure (SOP) [10] and opportunity to prepare and ask questions. Teams were blind to outcomes being measured.

\section{Interventions}

In both arms of the experiment, the clinical team performed PHEA on a mannequin, presented within a realistic pre-hospital clinical simulation [Additional file 2]. This included the decision to perform PHEA, and the performance of the procedure according to the services SOP. The preparation for PHEA involves establishing an equipment "kit dump" as well as the preparation and administration of drugs (Alfentanil (1 mg intravenously (IV)), Ketamine (2 mg/kg IV) and Rocuronium (1-1.2 mg/kg IV)). Correct placement of the tracheal tube (ETT) is confirmed by visualising it pass the vocal cords, by auscultation and by the measure of quantitative end-tidal capnography $\left(\mathrm{EtCO}_{2}\right)$, before securing it in place.

The fidelity of the simulation required it to be performed accurately in accordance with the ScotSTAR PHEA protocol [10], safely, in real time and with the retrieval consultant physician undertaking the role of intubator, and the HEMS paramedic the role of assistant.

The standard practice arm (unprepared), consisted of a drug bag containing all the required drug vials, syringes and labels to prepare for a PHEA, and a conventional airway bag holding all the required airway equipment. In this method, the "kit dump" and drugs are prepared according to the SOP after the decision to intubate has been made. The experimental method consisted of, equipment and drugs, optimally organised and prepared prior to the procedure being required (pre-prepared), having the kit dump pre-prepared within the airway bag, with individual items held securely in place (Fig. 2), and the drugs pre-prepared in labelled syringes (Fig. 3).

\section{Methods of measurements and outcomes measures}

Simulations were filmed, which allowed accurate measurement of outcomes and maintained blinding of participants to the outcomes being measured. Two reviewers independently analysed recordings and extracted study data from the video recording into a pre-prepared spreadsheet. Any discrepancy was resolved by consensus with a third independent reviewer who was blind to the study aims.

A full pre-hospital clinical simulation was simulated to reduce moulage artefact and mask the aspects of pre-hospital care that were being measured. Participants were blind to the study outcomes measured during the simulation (intervention time and error rate). Primary outcome was PHEA intervention time (minutes: seconds). Intervention time was defined as starting at the decision to perform PHEA and ending when correct ETT position was confirmed with the facilitator turning on the $\mathrm{EtCO}_{2}$ simulation software, in response to visualising chest inflation. Secondary outcomes included procedural errors, defined as an unintended/unexpected incident, which led, or could have led to harm. Errors were counted and classified according to Table 1. In 


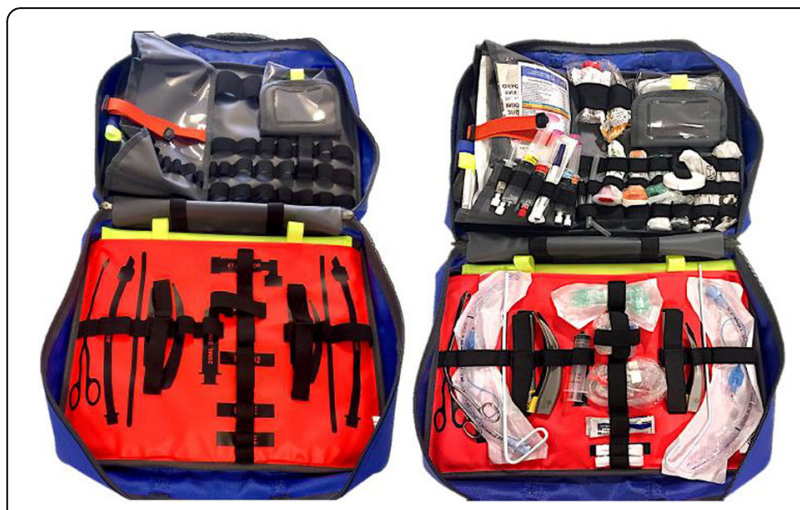

Fig. 2 The Adult SCRAM Bag. "The Adult SCRAM (Structured CRitical Airway Management) Bag is an Emergency Airway Bag which provides a structured reproduceable approach to airway management"[34]

addition, we assessed the degree of individual cognitive load (ICL), defined as the amount of cognitive work/energy required by the participant to complete the procedure, including the level of judgements/decisions needing to be made. ICL was measured using Visual analogue score (VAS). At the end of each simulation, participants were asked to indicate the magnitude of their perceived cognitive load during PHEA by marking a standard $100-\mathrm{mm}$ line appropriately $(0-\mathrm{mm}$ representing no cognitive load and $100-\mathrm{mm}$ representing maximal cognitive load). Visual analogue scores have been shown to be effective tools for measuring cognitive load [11, 12].

\section{Statistical analysis}

Statistical analysis was performed using SPSS 24.0 software (SPSS Inc., Chicago, IL, USA). Shapiro-Wilk test and normal-quartile plots were used to assess normality. Categorical data are reported as frequency (n) and percent (\%), and numerical data are reported as mean with Standard Deviation (SD) or median with Interquartile Range (IQR). Sample size was calculated using data from observation of prior EMRS practice: in ten consecutive standard PHEA's, the mean procedural time was 20:03

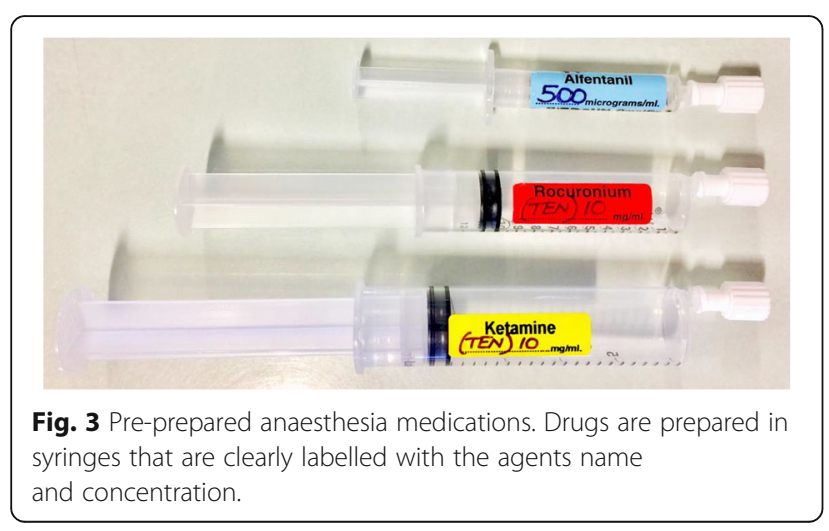

(3:26) minutes: seconds. A $20 \%$ reduction in procedural time was considered clinically significant. We determined that eleven simulations in each arm of the study were required for the paired $t$-test to have a $90 \%$ chance of detecting a difference in means of four minutes at a level of significance of 5\% (two-sided). We adjusted the sample size to twelve simulations in each arm to allow for any exclusions.

Parametric data were compared using the paired $t$-test and non-parametric data were compared using Wilcoxon matched-pair signed-rank test. An absolute (Mean Difference, MD, or Difference of Medians, DM) measure of intervention effect with 95\% Confidence Intervals (CI) was calculated for primary and secondary outcomes. A two-tailed $P$-value $<0.05$ was considered significant.

\section{Results}

Characteristics of study participants

Twenty-three simulations (11 in standard arm, 12 in experimental arm) were completed and the data included in this analysis. Characteristics of participating clinicians are described in Table 2. One simulation (standard arm) could not be completed due to operational demands. The outcomes of PHEA simulation using the two methods of equipment and drug preparation are presented in Table 3.

\section{Primary outcome: Intervention time}

Overall, the average intervention time was 16:13 (SD 5:17) minutes: seconds. Teams were able to perform PHEA significantly faster using the experimental method compared to standard practice (11:45 (SD 1:45) versus 20:59 (SD 3:13); MD 9:14 (95\% CI, 7:42 to 10:45) minutes: seconds; $\mathrm{p}=<0.001$ ) (Students $t$ ).

Group comparison demonstrated that slow working teams worked slowly in both methods, and faster working teams worked faster in both methods. Despite this, all teams were significantly quicker when using the experimental method. Pre-preparation of drugs resulted in the largest procedural time savings (Table 4).

\section{Secondary outcomes \\ Procedural errors}

Overall, 99 errors occurred during the 23 PHEA simulations (Table 5). Significantly fewer errors occurred when teams used the experimental method compared to standard practice: (0 (IQR: 0 to 2) versus 9 (IQR: 0 to 17); DM 9 (95\% CI, 4.5 to 12.8 ); $p=0.007$ ) (Wilcoxon matched-pair signed-rank).

\section{Cognitive load}

Cognitive load of the intubator was similar between groups (49.9 (SD 20.5) $\mathrm{mm}$ versus 49.4 (SD 20.5) $\mathrm{mm}$; MD 0.5 (95\% CI -16.7 to 17.8$) \mathrm{mm} ; p=0.945)$ (Students 
Table 1 Error Classifications

\begin{tabular}{|c|c|c|}
\hline Classification & Definitions & Examples \\
\hline Error & $\begin{array}{l}\text { Procedural error in the preparation or use of medications or } \\
\text { equipment with the potential to result in harm. }\end{array}$ & $\begin{array}{l}\text { Medication: } \\
\text { o Syringe containing anaesthesia medication labelled incorrectly } \\
\text { or not labelled } \\
\text { o Incorrect medication administered } \\
\text { o Incorrect dose administered } \\
\text { Equipment: } \\
\text { o Sharps injury } \\
\text { o Procedure performed not in accordance with SOP (i.e. checklist } \\
\text { not used, bougie not used) }\end{array}$ \\
\hline Lapse & $\begin{array}{l}\text { A failure to execute an action due to lapse in memory and a } \\
\text { routine behaviour being omitted. }\end{array}$ & $\begin{array}{l}\text { Medication preparation: } \\
\text { ○ Same needle used to draw up multiple medications } \\
\text { ○ No syringe cap } \\
\text { o Unsafe sharps management } \\
\text { Equipment preparation: } \\
\text { o Cuff of tracheal tube not checked } \\
\text { ○ Laryngoscope bulb operation not checked } \\
\text { ○ No bougie }\end{array}$ \\
\hline
\end{tabular}

t). However, the cognitive load of the intubator assistant was significantly reduced when using the experimental method compared to standard practice: (41.9 (SD 22.4) $\mathrm{mm}$ versus 68.7 (SD 24.8) $\mathrm{mm}$; MD 26.8 (95\% CI 9.8 to 43.8) $\mathrm{mm} ; p=0.006$ ) (Students $t$ ) (Fig. 4).

\section{Discussion}

Key findings

This study demonstrates the effectiveness of a system of pre-preparation of equipment and drugs, together with optimal ergonomic organisation of equipment, for PHEA. Our results show a significant and clinically meaningful reduction in 1) the time it takes to perform the procedure, 2) errors during procedure / or significantly safer performance, and 3) the cognitive load of operators.

When aiming for short scene times $(<30 \mathrm{~min})$ a reduction of 9:14 min is clinically meaningful. Two elements contributed to this: 1) the time to set up the equipment

Table 2 Baseline Characteristics of study participants

\begin{tabular}{lll}
\hline Characteristics & $\begin{array}{l}\text { Consultant } \\
\text { Physicians } \\
(n=12)\end{array}$ & $\begin{array}{l}\text { HEMS } \\
\text { Paramedics } \\
(n=12)\end{array}$ \\
\hline $\begin{array}{l}\text { Age (years) } \\
\text { Gender (male) }\end{array}$ & $12(34$ to 53) & 44 (35 to 49) \\
Background Speciality & $9(75)$ \\
$\begin{array}{l}\text { Emergency Medicine } \\
\text { Anaesthetists / Intensivist } \\
\text { HEMS Paramedic }\end{array}$ & $10(83)$ & $\mathrm{n} / \mathrm{a}$ \\
NHS Consultant Physician Experience & $9(2$ to 16) & $\mathrm{n} / \mathrm{a}$ \\
$\begin{array}{l}\text { (years) } \\
\text { Paramedic Experience (years) }\end{array}$ & $\mathrm{n} / \mathrm{a}$ & $12(100)$ \\
PHEA Experience (years) & $n / a$ & $13.5(8$ to 28) \\
\hline
\end{tabular}

*Median experience of HEMS paramedics as frontline ambulance paramedics "kit dump" and 2) the time to prepare the required drugs, which accounted for most of the time saved.

Most of the errors and safety-related incidents occurred during the preparation and labelling of drugs on scene. In one incident, the intubator assistant cut a finger while opening an ampoule, highlighting the risk of sustaining a sharps injury when working under pressure. In another, a Rocuronium syringe was not labelled, and then confused for another agent, highlighting the risk of a drug administration error. These errors and safety-related incidents were, however, eliminated by using pre-drawn-up drugs in labelled syringes, resulting in significantly safer operation.

The variance in procedural time was less in the experimental arm, which may suggest an improved workflow. By improving workflow, overall performance was enhanced, and cognitive load was reduced. However, realising where cognitive resilience within a team lies is an important consideration, especially when performing complex, high-risk interventions such as PHEA. In our study, the intubator assistant reported a significantly reduced CL in the experimental method, even lower than that of the intubator. This enables the team to utilise this resilience to their advantage, for example by maintaining the team's situational awareness "through" the intubator assistant to deliver safe, timely, effective, high quality care as a team.

\section{Time reduction}

The initial resuscitation and evaluation of critically injured or ill patients begins in the pre-hospital environment, and the care that they receive can have a major influence on subsequent outcome [13, 14]. Providing individualised, tailored care based on injury patterns, means that some patients may require specialised care, such as PHEA to optimize their clinical condition prior to transfer [15]. However, 
Table 3 Outcomes - PHEA simulation using standard practice and an experimental method of equipment and drug preparation

\begin{tabular}{|c|c|c|c|c|}
\hline Outcome & $\begin{array}{l}\text { Standard } \\
\text { Practice } \\
\text { (11 simulations) }\end{array}$ & $\begin{array}{l}\text { Experimental Method } \\
\text { (12 simulations) }\end{array}$ & $\begin{array}{l}\text { Mean Difference } \\
(95 \% \mathrm{Cl})\end{array}$ & $P$-value \\
\hline Intervention Time (min:sec) & 20:59 (3:13) & $11: 45(1: 45)$ & $9: 14(7: 42$ to $10: 45)$ & $<0.001$ \\
\hline Errors & $9(0$ to 17$)$ & $0(0$ to 2$)$ & $8.6(4.5 \text { to } 12.8)^{*}$ & 0.007 \\
\hline Cognitive Load Intubator (mm) & $49.9(20.5)$ & $49.4(20.5)$ & $0.5(-16.7$ to 17.8$)$ & 0.945 \\
\hline Cognitive Load Assistant (mm) & $68.7(24.8)$ & $41.9(22.4)$ & 26.8 (9.8 to 43.1$)$ & 0.006 \\
\hline
\end{tabular}

* Difference of Medians with $95 \% \mathrm{Cl}$

these interventions are known to increase time on scene, $[2,16]$ while the Association of Anaesthetists of Great Britain and Ireland stipulate that "every effort must be made to keep pre-hospital time to a minimum"[2]. As a service, we aim to spend time on scene wisely, and minimise time from incident to definitive care. To spend a large proportion of this time preparing for PHEA, while caring for a critically ill or injured patient, is not effective use of time.

Using the concept of "aggregation of marginal gains" [17] and breaking down the intervention (PHEA) into its core components, we identified the preparation phase of a procedure to be critical in determining both the safety of the procedure and the time it takes to perform PHEA. We were then able to demonstrate a significant time reduction in delivering the intervention. It was also clear that most of the preparation for this procedure could be done before the procedure became necessary, i.e. in controlled undisturbed conditions at base rather than on-scene with all the attendant competing demands on our attention and potential for distractions and interruptions. This time saved may be reflected in reducing scene times and time to definitive care. However, performing an intervention more quickly does not automatically mean that it is performed more safely.

\section{Error reduction}

Human error is an important problem in health care, contributing to a high instance of preventable medication errors [18-21]. Preparing drugs is a time-consuming process, requiring precision. Carrying out this critical task, while at the same time treating a critically injured patient in an uncontrolled pre-hospital environment, is far from desirable and inherently prone to error. Using standard practice of PHEA preparation, our study shows that $40 \%$ of on-scene time was spent preparing drugs for PHEA, and most of the errors that occurred arose during the preparation of drugs on scene. These included (Table 5): drug labelling errors, omission of labels, poor sharps management and inadvertent "syringe swaps", all of which can cause serious patient harm [20-22]. For example, routine practice is to prepare Rocuronium (100 mg) in a $10 \mathrm{ml}$ syringe, and Ketamine $(200 \mathrm{mg}$ ) in a $20 \mathrm{ml}$ syringe. In one observed error, Rocuronium (200 mg) was prepared in a $20 \mathrm{ml}$ syringe, and subsequently incorrectly labelled as Ketamine. This could have resulted in a neuromuscular drug being administered without prior anaesthesia, exposing the patient to harm.

Such incidents are "almost invariably judged to represent sub-standard care and litigation is almost invariably successful" [22, 23]. An anaesthetic practice review of 896 drug error reports that a large number of errors involve

Table 4 Component Times

\begin{tabular}{|c|c|c|c|c|}
\hline $\begin{array}{l}\text { Component } \\
\text { (min:sec) }\end{array}$ & $\begin{array}{l}\text { Standard Practice } \\
\text { (11 simulations) }\end{array}$ & $\begin{array}{l}\text { Experimental Method } \\
\text { (12 simulations) }\end{array}$ & $\begin{array}{l}\text { Mean Difference } \\
(95 \% \mathrm{Cl})\end{array}$ & $P$-value \\
\hline Equipment preparation & $\begin{array}{l}05: 01 \\
\text { (03:42 to } 06: 19)\end{array}$ & $\begin{array}{l}03: 57 \\
\text { (03:13 to } 04: 41)\end{array}$ & $\begin{array}{l}01: 04 \\
(-00: 18 \text { to } 02: 26)\end{array}$ & 0.114 \\
\hline Drug Preparation & $\begin{array}{l}08: 23 \\
\text { (06:43 to } 10: 03)\end{array}$ & $\begin{array}{l}00: 16 \\
(00: 07 \text { to } 00: 24)\end{array}$ & $\begin{array}{l}08: 07 \\
\text { (06:26 to 09:49) }\end{array}$ & $<0.001$ \\
\hline Checklist & $\begin{array}{l}\text { 03:50 } \\
\text { (03:02 to } 04: 38)\end{array}$ & $\begin{array}{l}03: 40 \\
\text { (03:12 to } 04: 08)\end{array}$ & $\begin{array}{l}00: 10 \\
(-00: 27 \text { to } 00: 47)\end{array}$ & 0.551 \\
\hline Drug Administration Including Onset Time & $\begin{array}{l}01: 36 \\
(01: 16 \text { to } 01: 57)\end{array}$ & $\begin{array}{l}01: 40 \\
(01: 31 \text { to } 01: 49)\end{array}$ & $\begin{array}{l}-00: 04 \\
(-00: 24 \text { to } 00: 17)\end{array}$ & 0.702 \\
\hline Tracheal Intubation & $\begin{array}{l}00: 42 \\
(00: 32 \text { to } 00: 51)\end{array}$ & $\begin{array}{l}00: 37 \\
(00: 32 \text { to } 00: 43)\end{array}$ & $\begin{array}{l}00: 04 \\
(-00: 07 \text { to } 00: 15)\end{array}$ & 0.407 \\
\hline Total Intervention time & $\begin{array}{l}20: 59 \\
(18: 49 \text { to } 23: 09)\end{array}$ & $\begin{array}{l}11: 45 \\
(10: 34 \text { to } 12: 56)\end{array}$ & $\begin{array}{l}09: 14 \\
(07: 42 \text { to } 10: 45)\end{array}$ & $<0.001$ \\
\hline
\end{tabular}

Data presented as mean $(95 \% \mathrm{Cl})$

Definitions of Pre-hospital Anaesthesia components [see Additional file 3] 
Table 5 Characteristics of procedural errors

\begin{tabular}{llll}
\hline Characteristics & $\begin{array}{l}\text { Standard } \\
\text { Practice }\end{array}$ & $\begin{array}{l}\text { Experimental } \\
\text { Method }\end{array}$ & $p$-value \\
\hline $\begin{array}{l}\text { Laps in medication } \\
\text { preparation }\end{array}$ & 41 & 0 & 0.007 \\
$\begin{array}{l}\text { Error in medication } \\
\text { preparation }\end{array}$ & 31 & 0 & 0.011 \\
$\begin{array}{l}\text { Laps in equipment } \\
\text { preparation }\end{array}$ & 23 & 2 & 0.0027 \\
$\begin{array}{l}\text { Error in equipment } \\
\text { preparation }\end{array}$ & 2 & 0 & 0.317 \\
\begin{tabular}{l} 
Total errors \\
\hline
\end{tabular} & 97 & 2 & 0.007 \\
\hline
\end{tabular}

drugs in similar sized syringes, along with drug preparation errors, which suggest that this is a frequently occurring incident. [24] In a systematic review of drug administration error prevention during anaesthesia, Jensen et al. recommends "drugs should be presented in prefilled syringes (where possible) rather than ampoules (either for emergency drugs or in general)" [25]. This is also supported by the Anaesthesia Patient Safety Foundation as part of a "new paradigm" to reduce the number of drug related errors, and improve patient safety [26].

Currently, there is wide variation in the way that pre-hospital services prepare drugs for PHEA, including using pharmacy-prepared drugs in pre-filled syringes, teams preparing the drugs at the start of the shift, drawing them up en route to an incident, and drawing them up on scene.

Syringes can be pre-prepared by the service or pharmacy. Individual services would need to consider the associated costs, waste, and shelf life of each method [Additional file 4]. A barrier to pre-prepared drugs maybe the additional cost of pre-prepared drugs or concerns over the risk of drug wastage. The additional cost may, however, be offset by the accompanying reduction in the frequency of errors in preparing intravenous drugs and, more importantly, the iatrogenic harm and human cost of such errors [22, 27]. Furthermore, the magnitude of the time reduction to administer the drugs for PHEA using pre-filled labelled syringes cannot be ignored.

\section{Reduction of cognitive load}

Cognitive load can affect human performance. The effect of human performance on the safe delivery of anaesthesia is widely recognised. Over $40 \%$ of adverse outcomes reported to the 4th National Audit Project (NAP4) [4] were attributed to human factors. "Cognitive resources, though limited, are under conscious control and can be directed from task to task as necessary" [28]. In the complex and unpredictable pre-hospital environment, the clinician is faced with additional load, beyond that of

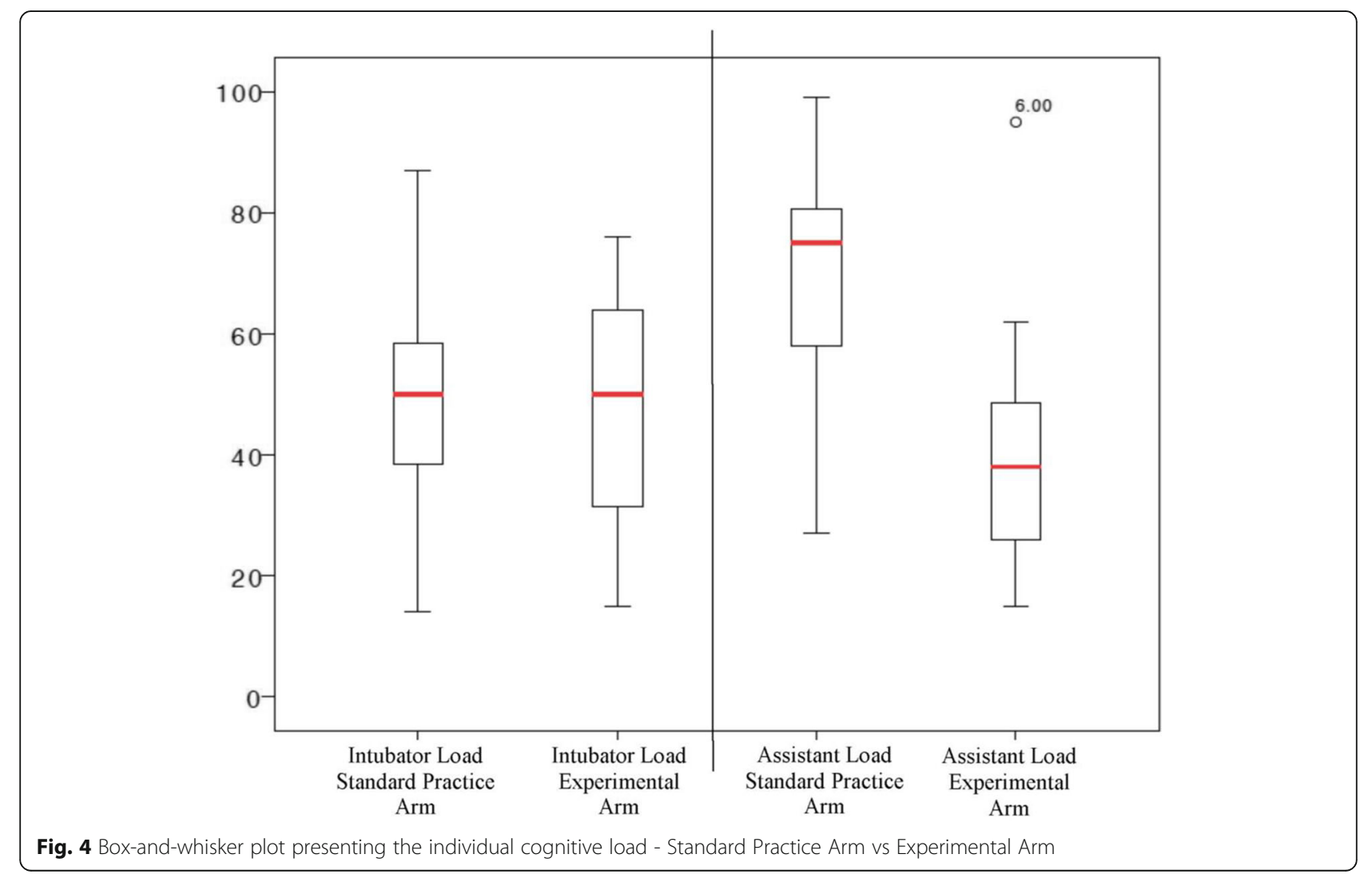


delivery of the PHEA. The cognitive demands of managing oneself, the team and the environment can exacerbate an escalating workload, risking plan continuation bias and cognitive overload [29]. This can compromise the delivery of safe, effective high quality care [30], as demonstrated in the seminal case of Elaine Bromley, an example of the considerable harm that can result from cognitive overload [31].

There are several ways of reducing cognitive load in critical situations, including the development of strategies such as briefings, flows (workflow patterns), and checklists and limiting the number of critical decisions that need to be made. The cognitive burden can potentially be further reduced by standardising the equipment and processes required for the intervention, for example by streamlining packaging or numbering various components sequentially. Such improvements could enhance patient safety by contributing to greater reliability, resilience and situational awareness [22, 4, 32].

There is a recognised relationship between workflow and cognitive load [28] and this can be influenced by the storage and presentation of equipment [33]. If the method of storing and presenting equipment for an intervention is designed to reflect more precisely the series and sequence of steps required for that intervention, the method itself becomes a useful "tool" for reducing the cognitive burden (see Fig. 2).

We hope the findings of this study will support a change in practice from on-scene PHEA drug and equipment preparation to pre-preparation.

We believe that the results of our study are generalisable to any pre-hospital situation where PHEA is being delivered, as factors such as time, safety and cognitive load are the same regardless of the model of pre-hospital care (physician/paramedic, nurse/paramedic, critical care paramedic/paramedic).

The strengths of this study include the realistic simulation of a pre-hospital scenario, allowing unbiased measurement of important aspects of PHEA, which would likely not be possible under real conditions.

Our study has several limitations. This experiment was done in a simulated setting, so the results may not replicate true clinical practice. However, the pre-hospital clinical simulation [Additional file 2] was piloted by clinicians not involved in the study, before the trial recruitment began, to ensure that the simulation was reproducible, straightforward, and that it recreated the clinical practice as closely as possible. It is likely that real pre-hospital cases would be even more complex than those simulated, and would result in even more errors.

The same pre-hospital clinical simulation was used in both methods of the trial which may have introduced exposure bias or training bias. A two-week washout period between the first and the second simulation was implemented to reduce this bias, and clinicians were blind to the outcomes being measured during the simulation. No difference in performance was seen either side of the washout period.

The VAS is used in a wide variety of populations and situations due to its adaptability and ease of use [35]. However, VAS is subjective, and some evidence exists that suggests that it lacks sensitivity and that risks of error exist in some subject groups [36]. We acknowledge that visual analogue scales have not been validated to measure cognitive load in this setting. Nevertheless, we feel that these simple tools are able to provide an unbiased meaningful message, that signal how cognitive resilience could be enhanced during this intervention.

A further limitation of this study is that only 23 of the 24 simulations were included into our analysis as one of the simulations (using standard practice) was incomplete and thus excluded. However, even if the quickest procedure time, across both groups, was input as the missing value, the procedural time using the experimental method remained significantly less.

\section{Conclusion}

Pre-preparation of PHEA drugs, and to a lesser extent the pre-preparation and organisation of PHEA equipment, significantly reduced procedural time and has the potential to reduce on-scene time substantially. In addition, pre-preparation of equipment and drugs resulted in safer performance of PHEA and reduced the cognitive load of the PHEA assistant.

\section{Additional Files}

\section{Additional file 1: Research Protocol (PDF $755 \mathrm{~kb}$ ) \\ Additional file 2: Pre-hospital clinical simulation (PDF $358 \mathrm{~kb}$ ) \\ Additional file 3: Definitions of Prehospital Anaesthesia components (DOCX $77 \mathrm{~kb}$ ) \\ Additional file 4: Pharmacy-prepared prefilled syringes cost and shelf life $[37,38]$ (DOCX 14 kb)}

\section{Abbreviations \\ CL: Cognitive load; DM: Difference of Medians; EMRS: Emergency Medical Retrieval Service; EtCO ${ }_{2}$ : End-tidal capnography; ETT: Tracheal tube; HEMS: Helicopter Emergency Services; ICL: Individual cognitive load; IQR: Interquartile Range; IV: Intravenously; MD: Mean Difference; mm: Milimeters; mm:ss: minutes: seconds; n: Frequency; PHEA: Pre-hospital Emergency Anaesthesia; SAS: Scottish Ambulance Service; ScotSTAR: Scotland's national Specialist Transport and Retrieval service; SCRAM : Structured CRitical Airway Management; SD: Standard Deviation; SHIL: Scottish Health Innovations; SOP: Standard operating procedure; VAS: Visual analogue score}

\section{Acknowledgements}

The authors would like to thank the HEMS paramedics from the Scottish Ambulance Service, and retrieval consultant physicians from the Emergency Medical Retrieval Service for their participation. As well as the EMRS Advanced Retrieval Practitioners for their assistance and support running the pre-hospital simulations. 


\section{Funding}

The cost of consumables used within this study was provided by Scottish Health Innovations Ltd. (SHIL). SHIL is a publicly owned private company limited by guarantee, wholly owned by Scottish Ministers and two health boards. Working in partnership with National Health Service (NHS) Scotland to support and develop innovative solutions which address a clearly identified healthcare need; with the proposed innovations being generated by NHS healthcare professionals.

\section{Availability of data and materials}

The authors declare that the data supporting the findings of this study are available within the article and its supplementary information files.

\section{Authors' contributions}

PS conceived the study and obtained research funding. PS, ZP, AC, CM and NS designed the protocol. ZP and PS supervised the conduct of the study and data collection. PS, DP and JP undertook recruitment of participating clinicians, filmed the simulations and analysed the video recordings including quality control. CM, ZP, AC provided statistical advice on study design and analysed the data. PS, CM, ZP and AC were responsible for data analysis and interpretation. PS drafted the manuscript, and all authors contributed substantially to its revision. PS takes responsibility for the paper as a whole. We confirm that the manuscript has been read and approved by all named authors and that there are no other persons who satisfied the criteria for authorship but are not listed. We further confirm that the order of authors listed in the manuscript has been approved by all of us. We confirm that we have given due consideration to the protection of intellectual property associated with this work and that there are no impediments to publication, including the timing of publication, with respect to intellectual property. In so doing we confirm that we have followed the regulations of our institutions concerning intellectual property.

\section{Authors' information}

1. PS. Dip IMC, MSc. HEMS Paramedic \& Retrieval Practitioner.

2. AC. MPH. Consultant in Emergency \& Retrieval Medicine.

3. CM. MBChB, MCEM. Clinical Research Fellow.

4. DP. BSc. Paramedic \& Retrieval Practitioner.

5. JP. BSC, PgC. Paramedic \& Advanced Retrieval Practitioner.

6. NS. BSC, MSc. Consultant Paramedic.

7. ZP. MBBCh, PhD. Honorary Clinical Lecturer.

\section{Ethics approval and consent to participate}

The study was reviewed and approved by Queen Mary, University of London Research Ethics Committee (QMREC1839a), Greater Glasgow and Clyde Clinical Research \& Development committee (GN16AE762), and the Scottish Ambulance Service. Written informed consent was obtained from participants prior to their participation.

\section{Consent for publication}

Written informed consent was obtained from all participants for the composite data collected in this study to be used for publication.

\section{Competing interests}

We wish to draw the attention of the Editor to the following facts which may be considered as potential conflicts of interest and financial contributions to this work. Authors, PS and NS are co-inventors of the $\mathrm{SCRAM}^{\oplus}$ bag (Structured CRitical Airway Management) which is an emergency airway bag used within this study. The SCRAM ${ }^{\oplus}$ bag is now a commercially available product and as such PS and NS receive a royalty. We confirm that the remaining authors have no known conflicts of interest associated with this publication.

\section{Publisher's Note}

Springer Nature remains neutral with regard to jurisdictional claims in published maps and institutional affiliations.

\section{Author details}

${ }^{1}$ Emergency Medical Retrieval Service, ScotSTAR, Scottish Ambulance Service, Glasgow, UK. ${ }^{2}$ Scottish Air Ambulance Division, Scottish Ambulance Service, Glasgow, UK. ${ }^{3}$ Royal Alexandra Hospital, Paisley, UK. ${ }^{4}$ ScotSTAR, Scottish Ambulance Service, Glasgow, UK. ${ }^{5}$ Scottish Ambulance Service, Clinical
Directorate, Edinburgh, UK. ${ }^{6}$ Centre for Trauma Sciences, Queen Mary, University of London, London, UK.

Received: 5 July 2018 Accepted: 12 September 2018

Published online: 21 September 2018

\section{References}

1. Brown J, Rosengart M, Forsythe R, Reynolds B, Gestring M, Hallinan W, et al. Not all prehospital time is equal: influence of scene time on mortality. J Trauma Acute Care Surg. 2016;81:93-100.

2. Lockey D, Crewdson K, Davies G, Jenkins B, Klein J, Laird C, et al. AAGBI: safer pre-hospital anaesthesia 2017. Anaesth. 2017;72:379-90.

3. Weingart S, Levitan R. Preoxygenation and prevention of desaturation during emergency airway management. Ann Emerg Med. 2012;59:165-75.

4. Cook T, Woodall N, Frerk C. Major complications of airway management in the UK: results of the fourth National Audit Project of the Royal College of Anaesthetists and the difficult airway society. Part 1: Anaesthesia. $\mathrm{Br} J$ Anaesth. 2011:106:617-31.

5. Cook T, Woodall N, Harper J, Benger J. Major complications of airway management in the UK: results of the fourth National Audit Project of the Royal College of Anaesthetists and the difficult airway society. Part 2: intensive care and emergency departments. Br J Anaesth. 2011;106:632-42.

6. Burgess M CK, Locky D.J, Perkins Z.B. Pre-hospital emergency Anaesthesia: an updated survey of UK practice with emphasis on the role of standardisation and checklists. Emerg Med J 2018 (in press). 2018

7. National Records of Scotland. Scotland's Population is Increasing, 2017. https://www.nrscotland.gov.uk/news/2017/scotlands-population-isincreasing. Accessed 20 Mar. 2018.

8. Emergency Medical Retrieval Service. Home, 2018. https://www. emrsscotland.org/. Accessed 20 Mar. 2018.

9. Great North Air Ambulance. Pre-hospital anaesthesia course, 2016. https:// www.greatnorthairambulance.co.uk/pha/. Accessed 20 Mar. 2018.

10. ScotSTAR. Standard operating procedure emergency Anaesthesia. Scottish ambulance service 2015

11. Gentili R, Rietschel J, Jaquess K, Lo L-C, Prevost C, Miller M, et al. Brain biomarkers based assessment of cognitive workload in pilots under various task demands. Conf Proc IEEE Eng Med Biol Soc. 2014: 5860-3.

12. DiDomenico AT. An investigation on subjective assessments of workload and postural stability under conditions of joint mental and physical demands: Virginia Tech; 2003.

13. Lockey DJ, Crewdson K, Lossius HM. Pre-hospital anaesthesia: the same but different. Br J Anaesth. 2014;113:211-9.

14. van der Velden MW, Ringburg AN, Bergs EA, Steyerberg EW, Patka P, Schipper IB. Pre-hospital interventions: time wasted or time saved? An observational cohort study of management in initial trauma care. Emerg Med J. 2008;25:444-9.

15. Harmsen AM, Giannakopoulos GF, Moerbeek PR, Jansma EP, Bonjer HJ, Bloemers FW. The influence of pre-hospital time on trauma patients outcome: a systematic review. Injury. 2015;46:602-9.

16. Gausche M, Lewis RJ, Stratton SJ, Haynes BE, Gunter CS, Goodrich SM, et al. Effect of out-of-hospital pediatric endotracheal intubation on survival and neurological outcome: a controlled clinical trial. JAMA. 2000;283:783-90.

17. Durrand J, Batterham A, Danjoux G. Pre-habilitation (i): aggregation of marginal gains. Anaesthesia. 2014;69:403-6.

18. Glavin R. Drug errors: consequences, mechanisms, and avoidance. $\mathrm{Br}$ Anaesth. 2010;105:76-82.

19. Stelfox HT, Palmisani S, Scurlock C, Orav EJ, Bates DW. The "to err is human" report and the patient safety literature. BMJ Quality and Safety in Health Care. 2006;15:174-8

20. Brennan TA, Leape LL, Laird NM, et al. Incidence of adverse events and negligence in hospitalized patients: results of the Harvard medical practice study I. N Engl J Med. 1991;324:370-6.

21. Leape LL, Brennan TA, Laird N, Lawthers AG, Localio AR, Barnes BA, et al. The nature of adverse events in hospitalized patients: results of the Harvard medical practice study II. N Engl J Med. 1991;324:377-84.

22. Pandit J, Andrade J, Bogod D, Hitchman J, Jonker W, Lucas N, et al. The 5th National Audit Project (NAP5) on accidental awareness during general anaesthesia: summary of main findings and risk factors. Br J Anaesth. 2014; 113:549-59. 
23. Cook T, Scott S, Mihai R. Litigation related to airway and respiratory complications of anaesthesia: an analysis of claims against the NHS in England 1995-2007. Anaesthesia. 2010;65:556-63.

24. Abeysekera A, Bergman IJ, Kluger MT, Short TG. Drug error in anaesthetic practice: a review of 896 reports from the Australian incident monitoring study database. Anaesthesia. 2005;60:220-7.

25. Jensen LS, Merry AF, Webster CS, Weller J, Larsson L. Evidence-based strategies for preventing drug administration errors during anaesthesia. Anaesthesia. 2004;59:493-4.

26. Anesthesia Patient Safety Foundation. Medication safety in the operating room: time for a new paradigm on line; 2015. http://apsf.org/resources/ med-safety/. Accessed 20 Mar. 2018.

27. Webster CS, Merry AF, Ducat CM. Safety, cost and predrawn emergency drugs. Anaesthesia. 2001;56:799-20.

28. Loukopoulos LD, Dismukes R, Barshi I. Cockpit interruptions and distractions: a line observation study. National Aeronautics and Space Administration 2001. https://human-factors.arc.nasa.gov/ihs/flightcognition/Publications/LL_ KD_ISAP01DOC.pdf. Accessed 30 May 2018.

29. Berman BA, Dismukes RK. Pressing the approach: a NASA study of 19 recent accidents yields a new perspective on pilot error. Aviat Saf World. 2006;206:28-33.

30. Lauria MJ, Gallo IA, Rush S, Brooks J, Spiegel R, Weingart SD. Psychological skills to improve emergency care providers' performance under stress. Ann Emerg Med. 2017;70:884-90.

31. Clinical Human Factors Group. Elaine Bromiley's story, 2010. http://chfg.org/ stories/elaine-bromileys-story/. Accessed 20 Mar. 2018.

32. Difficult Airway Society. Difficult Airway Trolley (DAT), 2017. https://www.das. uk.com/files/Difficult_airway_trolley_DAS.pdf. Accessed 20 mar. 2018.

33. Petrosoniak A, Gray A, Fan M, White K, McGowan M, Pinkney S, et al. MP036: trauma resuscitation using in-situ simulation team training (TRUST): a novel approach to latent safety threat identification in trauma care, vol. 18: CJEM Cambridge University Press; 2016. p. S78-9.

34. Swinton P. SCRAM System. 2018; https://www.shil.co.uk/uploads/tinymce/ Adult\%20SCRAM\%20Bag\%202.0.pdf. Accessed 21 Jul, 2018.

35. Huttunen $\mathrm{K}$, Keränen $\mathrm{H}$, Väyrynen $\mathrm{E}$, Pääkkönen $\mathrm{R}$, Leino T. Effect of cognitive load on speech prosody in aviation: evidence from military simulator flights. Appl Ergon. 2011:42:348-57.

36. Wewers ME, Lowe NK. A critical review of visual analogue scales in the measurement of clinical phenomena. Res Nurs Health. 1990;13:227-36

37. Pollock K. NHS greater Glasgow and Clyde pharmacy production unit. Hospital Pharmacy Stock System. Accessed 14 Aug. 2018.

38. National Institute for Health and Care Excellence (NICE). Guidance on intravenous infusions, 2018. https://bnf.nice.org.uk/guidance/guidance-onintravenous-infusions.html. Accessed 14 Aug. 2018.

Ready to submit your research? Choose BMC and benefit from:

- fast, convenient online submission

- thorough peer review by experienced researchers in your field

- rapid publication on acceptance

- support for research data, including large and complex data types

- gold Open Access which fosters wider collaboration and increased citations

- maximum visibility for your research: over $100 \mathrm{M}$ website views per year

At BMC, research is always in progress.

Learn more biomedcentral.com/submissions 